\title{
Partial cystectomy in young male for a urachal tumor masquerading a bladder leiomyoma
}

\author{
Maurizio Sodo ${ }^{1}$, Lorenzo Spirito ${ }^{2}$, Roberto La Rocca ${ }^{2}$, Umberto Bracale ${ }^{1}$, Ciro Imbimbo ${ }^{2}$ \\ ${ }^{1}$ Department of Surgery, University of Naples Federico II, Naples, Italy; \\ ${ }^{2}$ Department of Urology, University of Naples Federico II, Naples, Italy.
}

\begin{abstract}
Summary Leiomyoma of the bladder is a very rare disorder that accounts for $0.43 \%$ of all bladder neoplasms. Although the pathophysiology of the bladder leiomyoma is unknown, there are some theories on it. The patients can be asymptomatic; when present, clinical symptoms (lower urinary tract symptoms andor hematuria), are associated with tumor size and location. For diagnosis, imaging plays an important role: ultrasound, computed tomography (CT) scan and magnetic resonance imaging (MRI) are the examinations most frequently performed. Treatment consists of surgical removal of the tumor, and the prognosis is excellent.
\end{abstract}

KEY WORDS: Bladder; Leiomyoma; Partial cystectomy.

Submitted 10 September 2018; Accepted 27 September 2018

\section{Case report}

Herein we present a case of leiomyoma of the bladder in young male patient who has been successfully managed with a partial cystectomy using a laparoscopic approach. A 33 year old male with a history of persistent thymus gland was referred to our urology clinic for pelvic pain, urgency and dysuria. No hematuria nor history of weight loss were noticed. His physical examination was unremarkable. Blood work and urine test were within normal limits.

An ultrasound of the abdomen and pelvis revealed no hydronephrosis nor masses of the upper tract. Although only partially filled, the bladder revealed a mass.

A Computed Tomography (CT) scan and Magnetic Resonance Imaging (MRI) confirmed the presence of an upper side bladder tumor (60 mm x $58 \mathrm{~mm}$ x $47 \mathrm{~mm}$ ) located along the urachus course without any evidence of distant metastasis but with an increased fat density around the mass (Figure 1A-B). The lesion appeared undissociated from the bladder wall.

The patient underwent a laparoscopic partial cystectomy and urachus removal with a margin of normal tissue by a 3 trocars technique. The bladder was closed in 2 layers and the peritoneum was then closed.

The patient had an uneventful post operative period and a Foley's catheter was left for 7 days. The mass measured grossly $6 \times 5 \times 4 \mathrm{~cm}$ in its greatest dimensions with a smooth surface and hard consistency. A microscopic description revealed typical features of a leiomyoma tumour: positive for Actina and negative for CD117 and
Protein S 100 (Figure 2A-B). We performed follow up with ultrasonography and CT scan and at 3 and 9 months. CT scans were negative. We evaluated bladder capacity with flowmetry at 3 and 9 months. Peak and average flows were $21 \mathrm{ml} / \mathrm{min}$ and $10 \mathrm{mil} / \mathrm{min}$. Voided volume was 198 $\mathrm{ml}$ at 3 months and at 9 months was increased at $296 \mathrm{ml}$.

\section{Figure 1.}

A. Large, hard tissue density mass arising from the urachus. The mass is not dissociable by the bladder wall at computed tomography.

B. High signal intensity mass on T1 magnetic resonance image.


Figure 2.

A. Microscopic description revealed typical features of leiomyoma, positive for Actina.

B. Microscopic description revealed typical features of leiomyoma, negative for CD117 and Protein S 100.

A.

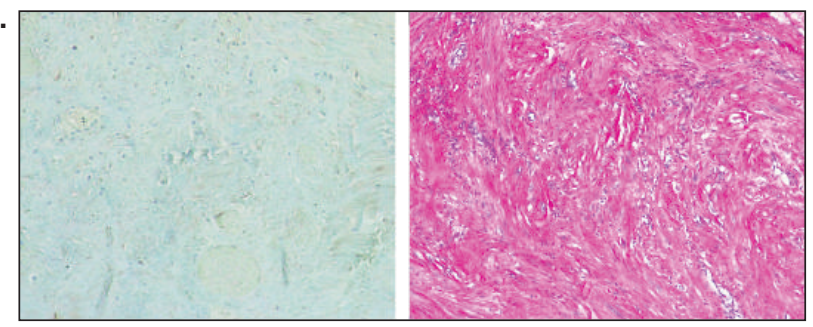

\section{Discussion}

In our case, ultrasonography showed a well-defined, encapsulated, lobulated mass of the bladder with a homogeneous and solid aspect, findings that were also supported by CT. While CT scans can identify the accu- 
rate location of leiomyoma tumors, they are not adequate to distinguish the solidity of the lesion. Therefore, ultrasonography should be performed in indeterminate lesions (as seen in CT) (2).

Magnetic resonance imaging (MRI) can distinguish between mesenchymal and transitional cell tumors (3). Leiomyomas have intermediate signal intensity on T1weighted images, while $\mathrm{T} 2$ - weighted images show a low signal intensity (4) but a definitive diagnosis is ultimately made by the histopathological examination.

Management of leiomyomas is mainly guided by the size and location of the tumor (5). Treatment options have ranged from enucleation, partial cystectomy and even, though rarely, radical cystectomy.

Leiomyomas occur throughout the genitourinary tract, which are most commonly found in the renal capsule. Most bladder tumors are derived from urothelial cells, while non-epithelial tumors of the bladder, particularly leiomyomas, are relatively rare, comprising of about $0.43 \%$ of all bladder tumors. In their review of 37 patients Goluboff et al. reported a preponderance of leiomyoma tumours in women $(76 \%)$ in their third through sixth decades (59\%) with a mean age of 44 years (5). In another series (6) all patients were women, with a mean age of 43.6 years, consistent with the report of Guluboff et al.

Bladder leiomyomas are known to produce symptoms, dependent primarily on their location and secondarily on their size.

Goluboff et al. demonstrated that patients most commonly presented with obstructive urinary symptoms (49\%), irritative symptoms (38\%), hematuria (11\%) or flank pain (13\%), while 19\% were asymptomatic. In another large series published by Park et al. (6), irritative symptoms occurred most frequently (37.7\%), followed by obstructive urinary symptoms $(31.1 \%)$, hematuria (24.6\%) and abdominal bulge or pain (14.8\%). Bladder leiomyoma are classified into three groups according to the histological location of tumor: endovesical, intramural and extravesical; which account for 63\%,7\%, and $30 \%$, respectively (6).

Traditionally, bladder leiomyomas have been treated by surgical resection. The tumor size, extent, and location and the involvement of the sphincter or ureter determine the route of resection. Small endovesical tumors can be managed with transurethral resection (TUR) and fulguration. Larger endovesical, intramural or extravesical tumors can best be managed with segmental resection or partial cystectomy (7). Transvaginal excision and laparoscopic partial cystectomy excision have all been successfully used for bladder leiomyoma removal $(8,9)$.

Some investigators have suggested that surgical removal should be reserved for symptomatic tumors. They assert that asymptomatic patients with a high probability of a leiomyoma as detected from the imaging, biopsy and cystoscopy evaluations can be followed up without invasive surgery due to the histologic similarities of bladder and uterine leiomyomas and no reported malignant transformation of bladder leiomyoma. However, bladder leiomyomas often mimic malignant lesions and, depending on the location, can often be diagnosed only after surgical removal. Furthermore, surgeons are familiar with performing TUR or partial cystectomy and therefore surgical resection is thought to be necessary for both confirmation of the diagnosis and definitive treatment.

Because the prognosis of patients with a leiomyoma tumor after surgical treatment is excellent and no malignant transformation has been reported to-date, a followup is not considered necessary unless urinary tract symptoms occur.

\section{Conclusions}

Leiomyoma of the urinary bladder is a rare disorder, comprising of about $0.43 \%$ of all bladder tumors. The tumor size, rather than the location, appears to affect the nature of symptoms. Successful treatment is up to surgeon, using varying surgical approaches, and the prognosis is good after complete resection.

\section{REFERENCES}

1. Cornella JL, Larson TR, Lee RA, et al. Leiomyoma of the female urethra and bladder: report of twenty-three patients and review of the literature. Am. J. Obstet. Gynecol. 1997; 176:1278-1285.

2. Illescas FF, Baker ME, Weinerth JL Bladder leiomyoma: advantages of sonography over computed tomography. Urol Radiol. 1986; $8: 216-218$

3. Sundaram CP, Rawal A., Saltzman B. Characteristics of bladder leiomyoma as noted on magnetic resonance imaging. Urology. 1998; 52:1142-1143.

4. Maya MM, Slywotzky C. Urinary bladder leiomyoma: magnetic resonance imaging findings. Urol Radiol. 1992; 14:197-199.

5. Goluboff ET, O'Toole K, Sawczuk IS. Leiomyoma of bladder: report of case and review of literature. Urology. 1994; 43:238-41.

6. Park JW, Jeong BC, Seo SI, et al. Leiomyoma of the urinary bladder: a series of nine cases and review of the literature. Urology. 2010; 76:1425-9.

8. Kanno K, Andou M, Yanai S, et al. Total laparoscopic treatment with cystotomy for intramural bladder leiomyoma. J Minim Invasive Gynecol. 2018; 25:14-15.

9. Yin FF, Wang N, Wang YL, et al. Transvaginal resection of a bladder leiomyoma misdiagnosed with a vaginal mass: a case report and literature review. Case Rep Obstet Gynecol. 2015; 2015:981843.

\author{
Correspondence \\ Maurizio Sodo, MD \\ maurizio Sodo sodo@unina.it \\ Umberto Bracale, $M D$ \\ bracale@unina.it \\ Department of Surgery, University of Naples Federico II, Naples (Italy)
}

Lorenzo Spirito, MD (Corresponding Author)

lorenzospirito@msn.com

Roberto La Rocca, MF

robertolarocca87@gmail.com

Ciro Imbimbo, MD

cimbimbo@unina.it

Department of Urology, University of Naples Federico II

Via S. Pansini 5, 80131 Naples (Italy) 